\title{
Asymptotics for General Nonstationary Fractionally Integrated Processes without Prehistoric Influence
}

QIYING WANG ${ }^{\dagger}$

qiying@maths.anu.edu.au CMA, School of Mathematical Science, The Australian National University, ACT 0200, Australia

YAN-XIA LIN AND CHANDRA GULATI

School of Mathematics and Applied Statistics, University of Wollongong, Wollongong, NSW 2522, Australia

Abstract. This paper derives a functional limit theorem for general nonstationary fractionally integrated processes having no influence from prehistory. Asymptotic distributions of sample autocovariances and sample autocorrelations based on these processes are also investigated. The problem arises naturally in discussing fractionally integrated processes when the processes starts at a given initial date.

Keywords: Functional limit theorem, fractional processes, sample autocovariances and sample autocorrelations.

\section{Introduction}

In recent times, there has been increasing interest in discussing the general fractional process $\left\{X_{t}\right\}$ defined by

$$
(1-B)^{d} X_{t}=u_{t}, \quad u_{t}=\sum_{j=0}^{\infty} \psi_{j} \epsilon_{t-j}, \quad t=1,2, \cdots,
$$

where $d>-1 / 2$ and $\epsilon_{t}$ are i.i.d. random variables with zero mean and finite variance. The $u_{t}$ are taken to be summable linear processes, i.e., we assume $\sum_{j=0}^{\infty}\left|\psi_{j}\right|<\infty$. B is a backshift operator, and the fractional difference operator $(1-B)^{\gamma}$ is defined by its Maclaurin series (by its binomial expansion, if $\gamma$ is an integer):

$$
(1-B)^{\gamma}=\sum_{j=0}^{\infty} \frac{\Gamma(-\gamma+j)}{\Gamma(-\gamma) \Gamma(j+1)} B^{j}
$$

$\dagger$ Requests for reprints should be sent to Qiying Wang,CMA, School of Mathematical Science, The Australian National University, ACT 0200, Australia. 
where $\Gamma(z)=\int_{0}^{\infty} s^{z-1} e^{-s} d s$, if $z>0 ; \Gamma(z)=\infty$ if $z=0$. If $z<0$, $\Gamma(z)$ is defined by the recursion formula $z \Gamma(z)=\Gamma(z+1)$. Thus $X_{t}$ can be expressed as

$$
\sum_{j=0}^{\infty} \frac{\Gamma(-d+j)}{\Gamma(-d) \Gamma(j+1)} X_{t-j}=u_{t}, \quad t=1,2, \cdots, \quad \text { if } d \neq 0,1,2, \cdots ;
$$

and

$$
\sum_{j=0}^{d}(-1)^{j} \frac{d !}{j !(d-j) !} X_{t-j}=u_{t}, \quad t=1,2, \cdots, \quad \text { if } d=0,1,2, \cdots,
$$

which depends on the "prehistoric influence":

$$
\sum_{j=t}^{\infty} \frac{\Gamma(-d+j)}{\Gamma(-d) \Gamma(j+1)} X_{t-j} \quad \text { or } \quad \sum_{j=t}^{d}(-1)^{j} \frac{d !}{j !(d-j) !} X_{t-j}
$$

In practice, if we ignore the value of $X_{t}, t \leq 0$, by assuming $X_{t}=0$, the process $X_{t}$ defined by (1) is then a special case of the process $Z_{t}$ (see Appendix 2) defined by

$$
Z_{t}=\sum_{k=0}^{t-1} c_{k}^{(d)} u_{t-k}, \quad u_{t}=\sum_{j=0}^{\infty} \psi_{j} \epsilon_{t-j}, \quad t=1,2, \cdots,
$$

where $c_{0}^{(0)}=1, c_{k}^{(0)}=0, k \geq 1 ; c_{k}^{(\alpha)}=\Gamma(k+\alpha) /\{\Gamma(\alpha) \Gamma(k+1)\}, k \geq 0$ and $\alpha \neq 0,-1, \cdots$.

Asymptotics of the process $Z_{t}$ were first investigated in Aknom and Gourieroux [1] with $d>1 / 2$ and $u_{t}=\epsilon_{t}$ under the condition $E\left|\epsilon_{0}\right|^{r}<$ $\infty$, where $r>\max \{2,2 /(2 d-1)\}$. The results of Aknom and Gourieroux [1] were extended to the multivariate case by Marinucci and Robinson [12] without any improvements in the moment conditions. Recently, Tanaka [16] discussed weak convergence for more general processes $Z_{t}$ where the $\psi_{k}$ satisfy $\sum_{k=0}^{\infty} k\left|\psi_{k}\right|<\infty$. However, the proof of Tanaka [16] depends on the functional limit theorem for martingale differences. Unfortunately, the process $Z_{t}$ itself (even with $u_{t}=\epsilon_{t}$ ) is not a martingale. Therefore, the proof of Tanaka [16] is not applicable in this case. In this paper, we give a different proof of the functional limit theorem for the process $Z_{[n t]}$. This proof shows that the main results given by Tanaka [16] still hold. Furthermore, we establish a similar result for a more general model under the weak moment condition $E\left|\epsilon_{0}\right|^{\max \{2,2 /(2 d-1)\}}<\infty$. It should be pointed out that the limit process of $Z_{[n t]} / \operatorname{Var}^{1 / 2}\left(Z_{n}\right)$ is different from those established for 
general fractional processes (cf. Wang, Lin and Gulati [17]) because of the "prehistoric influence".

There are four sections in this paper. The main results on functional limit theorem are given in Section 2. The study of the asymptotics of sample autocovariances and sample autocorrelations based on the process $Z_{t}$ is in Section 3. These results do not appear in the literature. We conclude in Section 4. Finally in the Appendices, we give the proof of Theorem 1, and a complementary proposition which shows that the process $X_{t}$ defined by (1) with $X_{t}=0, t \leq 0$, is a special case of the process $Z_{t}$ defined by (5).

Throughout this paper, we denote positive constants by $C$ with or without subscript, which might have different values in different places. A "type II" fractional Brownian motion $B_{d}(t), d>1 / 2$, is defined as

$$
B_{d}(0)=0, \quad B_{d}(t)=\int_{0}^{t}(t-s)^{d-1} d W(s), \quad 0 \leq t \leq 1,
$$

where $W(t)$ is a standard Brownian motion. Comparison between "type I" and "type II" fractional Brownian motions can be found in Marinucci and Robinson [11].

\section{Main Results}

In this section, a functional limit theorem is presented. Its proof will be given in Appendix 1. The most interesting part of this section is given in two corollaries.

Theorem 1 Let $Z_{t}$ satisfy (5) with

$$
\sum_{j=0}^{\infty}\left|\psi_{j}\right|<\infty \quad \text { and } \quad b_{\psi} \equiv \sum_{j=0}^{\infty} \psi_{j} \neq 0 .
$$

Then, for $d \geq 1$,

$$
\frac{1}{n^{d-1 / 2}} Z_{[n t]} \Rightarrow \kappa_{1}(d) B_{d}(t), \quad 0 \leq t \leq 1
$$

where $\kappa_{1}^{2}(d)=b_{\psi}^{2} \sigma^{2} / \Gamma^{2}(d)$ and $B_{d}(t)$ is a "type II" fractional Brownian motion. If, in addition, $E\left|\epsilon_{0}\right|^{2 /(2 d-1)}<\infty$ and $\sum_{k=0}^{\infty} k\left|\psi_{k}\right|<\infty$, then (7) still holds for $1 / 2<d<1$.

As a direct consequence of Theorem 1 and the continuous mapping theorem, the following corollary gives the asymptotic distribution of the partial sum process of the process $Z_{t}$, which will be useful in discussing the asymptotics of sample autocovariances and sample autocorrelations based on the process $Z_{t}$. 
Corollary 1 Let $Z_{t}$ satisfy (5), where $\psi_{j}$ satisfy (6). Then,

$$
\begin{aligned}
\frac{1}{n^{d+1 / 2}} \sum_{j=1}^{[n t]} Z_{j} & \Rightarrow \kappa_{1}(d+1) B_{d+1}(t), \text { for } d>1 / 2, \\
\frac{1}{n^{2 d}} \sum_{j=1}^{[n t]} Z_{j}^{2} & \Rightarrow \kappa_{1}^{2}(d) \int_{0}^{t} B_{d}^{2}(s) d s, \text { for } d \geq 1, \\
\frac{1}{n^{d-1 / 2}} \sum_{j=1}^{n-k}\left(Z_{j+k}-Z_{j}\right) & \Rightarrow k \kappa_{1}(d) B_{d}(1), \quad \text { for } d \geq 1,
\end{aligned}
$$

where $0 \leq t \leq 1, \kappa_{1}(d)$ is defined as in Theorem 1 and $k$ is a fixed integer. If, in addition, $E\left|\epsilon_{0}\right|^{2 /(2 d-1)}<\infty$ and $\sum_{k=0}^{\infty} k\left|\psi_{k}\right|<\infty$, then (9) and (10) still hold for $1 / 2<d<1$.

Proof: Recalling $c_{j}^{(\alpha)}=\Gamma(j+\alpha) /\{\Gamma(\alpha) \Gamma(j+1)\}$ and $\Gamma(1+\alpha)=\alpha \Gamma(\alpha)$, we have that, for any integer $m \geq 1$ and $\alpha \neq 0,-1,-2, \cdots$,

$$
\sum_{j=0}^{m} c_{j}^{(\alpha)}=1+\frac{1}{\alpha \Gamma(\alpha)}\left[\frac{\Gamma(1+m+\alpha)}{\Gamma(1+m)}-\frac{\Gamma(1+\alpha)}{\Gamma(1)}\right]=c_{m}^{(1+\alpha)}
$$

(see Sowell [15]). This equality implies that, for $d>1 / 2$,

$$
\sum_{j=1}^{[n t]} Z_{j}=\sum_{j=1}^{[n t]} \sum_{k=1}^{j} c_{j-k}^{(d)} u_{k}=\sum_{k=1}^{[n t]} u_{k} c_{[n t]-k}^{(1+d)}=\sum_{k=0}^{[n t]-1} c_{k}^{(1+d)} u_{[n t]-k}
$$

By using Theorem 1 with $1+d$, we obtain the desired (8).

To prove (9), we note that $\left(\sum_{j=1}^{[n t]} Z_{j}^{2}\right) / n^{2 d}=\int_{0}^{1}\left(Z_{[n s]} / n^{d-1 / 2}\right)^{2} d s$ (let $\sum_{i=1}^{[n s]} Z_{i}=0$ if $\left.s<1 / n\right)$ and then use the continuous mapping theorem.

Using Theorem 1 , the continuous mapping theorem and $\sum_{j=1}^{n-k}\left(Z_{j+k}-\right.$ $\left.Z_{j}\right)=\sum_{j=n-k+1}^{n} Z_{j}-\sum_{j=1}^{k} Z_{j}$, (10) follows easily. This completes the proof of Corollary 1.

In the next corollary, we consider the asymptotics for general stationary and general nonstationary fractionally integrated processes without prehistoric influence.

Corollary 2 Let $\alpha>-1 / 2$. Consider $X_{t}$ satisfying that $(1-B)^{\alpha} X_{t}=$ $u_{t}$, with $u_{t}=\sum_{k=0}^{\infty} \psi_{k} \epsilon_{t-k}$ for $t=1,2, \cdots ; X_{t}=0$, for $t \leq 0$, where $\sum_{k=0}^{\infty}\left|\psi_{k}\right|<\infty$ and $b_{\psi}=\sum_{k=0}^{\infty} \psi_{k} \neq 0$. Then, for $\alpha \geq 0$,

$$
\frac{1}{n^{1 / 2+\alpha}} \sum_{j=1}^{[n t]} X_{j} \Rightarrow \kappa_{2}(\alpha) \int_{0}^{t}(t-s)^{\alpha} d W(s), \quad 0 \leq t \leq 1,
$$


where $\kappa_{2}^{2}(\alpha)=b_{\psi}^{2} \sigma^{2} / \Gamma^{2}(1+\alpha)$. If, in addition, $E\left|\epsilon_{0}\right|^{2 /(2 \alpha+1)}<\infty$ and $\sum_{k=0}^{\infty} k\left|\psi_{k}\right|<\infty$, then (12) still holds for $-1 / 2<\alpha<0$.

Proof: It can be shown (see Appendix 2) that the process $X_{t}$ defined by Corollary 2 can be rewritten as $X_{t}=\sum_{k=0}^{t-1} c_{k}^{(\alpha)} u_{t-k}$, where $c_{k}^{(\alpha)}$ is defined as in (5). If $\alpha=0$, then $X_{t}=u_{t}$ and the result is obvious by using Theorem 1 with $d=1$. If $\alpha \neq 0$ and $\alpha>-1 / 2$, similar to (11), we obtain that $\sum_{j=1}^{[n t]} X_{j}=\sum_{k=0}^{[n t]-1} c_{k}^{(1+\alpha)} u_{[n t]-k}$. Since $1+\alpha>1 / 2$ when $\alpha>-1 / 2$, the results follow from Theorem 1 with $d=1+\alpha$. This completes the proof of Corollary 2.

\section{Sample Autocovariances and Sample Autocorrelations}

Let $Z_{1}, \cdots, Z_{n}$ be a sample drawn from a stationary process $\left\{Z_{t}\right\}$ with mean $\mu$, and define the lag- $k$ autocovariance and autocorrelation by $r_{k}=$ $E\left(Z_{1}-\mu\right)\left(Z_{1+k}-\mu\right)$ and $\rho_{k}=r_{k} / \operatorname{var}\left(Z_{1}\right)$. Usually, $r_{k}$ and $\rho_{k}$ are estimated through $\widehat{r}_{k}=\left\{\sum_{t=1}^{n-k}\left(Z_{t}-\bar{Z}\right)\left(Z_{t+k}-\bar{Z}\right)\right\} / n$ and $\widehat{\rho}_{k}=\widehat{r}_{k} / \widehat{r}_{0}$, where $\bar{Z}=$ $(1 / n) \sum_{t=1}^{n} Z_{t}$ is the sample mean.

In order to gain insight into the dependence of the process $\left\{Z_{t}\right\}$, the behaviours of the sample autocorrelations $\widehat{\rho}_{k}$ have been extensively studied in the literature under different sets of assumptions, for instance, inference for $\widehat{\rho}_{k}$ when the $Z_{t}$ is a linear process with i.i.d. innovations. Particularly, if $Z_{t}=\sum_{j=0}^{\infty} \psi_{j} \epsilon_{t-j}, t=1,2, \cdots$, where $\sum_{j=0}^{\infty} j^{1 / 2} \psi_{j}^{2}<\infty$, then the distributions of the first $k$ sample autocorrelations are asymptotically normal with mean $\left(\rho_{1}, \rho_{2}, \cdots, \rho_{k}\right)$ and covariance matrix $n^{-1} W$, where the $(i, j)$ th element of $W$ is given by $w_{i, j}=\sum_{r=1}^{\infty}\left\{\rho_{r+i}+\rho_{r-i}-2 \rho_{r} \rho_{i}\right\}\left\{\rho_{r+j}+\right.$ $\left.\rho_{r-j}-2 \rho_{r} \rho_{j}\right\}$ (for details, see Hannan and Heyde, [4]). With increasing generality, for further results on the asymptotics of $\widehat{r}_{k}$ and $\widehat{\rho}_{k}$ when the process $Z_{t}$ is a stationary or a nonstationary fractional process, we refer to Hasza [7], Newbold and Agiakloglou [13], Hosking [8], Bierens [2] as well as Hassler [5], [6].

In this section, we investigate the asymptotics of $\widehat{r}_{k}$ and $\widehat{\rho}_{k}$ when the process is $Z_{t}$ defined by (5). 
Theorem 2 Let $Z_{t}$ satisfy (5), where $d \geq 1$ and the $\psi_{j}$ satisfy (6). Then,

$$
\begin{aligned}
n^{1-2 d} \widehat{r}_{k} & \Rightarrow \kappa_{1}^{2}(d) \widetilde{B_{d}}(1) ; \\
\frac{n}{k}\left(\widehat{\rho}_{k}-1\right) & \Rightarrow-\frac{1}{2 \widetilde{B_{d}}(1)}\left\{\frac{B_{d+1}^{2}(1)}{d^{2}}+\left(B_{d}(1)-\frac{1}{d} B_{d+1}(1)\right)^{2}\right\}, \text { if } d \geq 1 \\
& \Rightarrow-\frac{1}{2 \widetilde{B_{d}}(1)}\left\{B_{d+1}^{2}(1)+\left(B_{d}(1)-B_{d+1}(1)\right)^{2}\right. \\
& \left.-\frac{1}{k b_{\psi}^{2} \sigma^{2}} E\left(\sum_{j=1}^{k} u_{j}\right)^{2}\right\}, \text { if } d=1,
\end{aligned}
$$

for any fixed integer number $k$, where $\kappa_{1}(d)$ is defined as in Theorem 1 and $\widetilde{B_{d}}(1)=\int_{0}^{1} B_{d}^{2}(t) d t-B_{d+1}^{2}(1) / d^{2}$.

Theorem 3 Let $Z_{t}$ satisfy (5). Assume that $E\left|\epsilon_{0}\right|^{2 /(2 d-1)}<\infty, \sum_{k=0}^{\infty} k\left|\psi_{k}\right|$ $<\infty$ and $b_{\psi} \equiv \sum_{k=0}^{\infty} \psi_{k} \neq 0$. Then, for any fixed integer number $k$ and $1 / 2<d<1$, (13) still holds and

$$
n^{-1+2 d}\left(\widehat{\rho}_{k}-1\right) \Rightarrow-\frac{I_{k}}{2 \kappa_{1}^{2}(d) \widetilde{B_{d}}(1)},
$$

where $\kappa_{1}(d), \widetilde{B_{d}}(1)$ are defined as in Theorem $2, c_{l}^{(d)}$ is as in (5) $\left(\right.$ let $c_{j}^{(d)}=0$ if $j<0)$ and $I_{k}=E\left(\sum_{l=0}^{\infty}\left(c_{l}^{(d)}-c_{l-1}^{(d)}\right) \sum_{i=1}^{k} u_{i-l}\right)^{2}$.

By noting that $\sum_{t=1}^{n-k}\left(Z_{t}-\bar{Z}\right)\left(Z_{t+k}-\bar{Z}\right)$ can be expressed as

$$
\sum_{t=1}^{n-k} Z_{t}\left(Z_{t+k}-Z_{t}\right)-\bar{Z} \sum_{t=1}^{n-k}\left(Z_{t+k}-Z_{t}\right)+\sum_{t=1}^{n-k}\left(Z_{t}-\bar{Z}\right)^{2},
$$

the proofs of Theorems 2- 3 follow easily from Corollary 1 , the continuous mapping theorem and the following proposition. We omit details.

Proposition 1 Let $Z_{t}$ satisfy (5), where $\psi_{j}$ satisfy (6). Then,

$$
\begin{aligned}
\frac{1}{n} \sum_{j=1}^{n-k}\left(Z_{j+k}-Z_{j}\right)^{2}-I_{k} & =o_{P}(1), \text { for } 1 / 2<d<1 ; \\
\frac{1}{n} \sum_{j=1}^{n-k}\left(Z_{j+k}-Z_{j}\right)^{2} & \rightarrow \text { a.s. } E\left(\sum_{j=1}^{k} u_{j}\right)^{2}, \text { for } d=1 ; \\
\frac{1}{n^{-1+2 d}} \sum_{j=1}^{n-k}\left(Z_{j+k}-Z_{j}\right)^{2} & =\quad o_{P}(1), \text { for } d>1 .
\end{aligned}
$$


for any fixed integer number $k$, where $I_{k}$ is defined as in Theorem 3.

Proof: $Z_{j+k}-Z_{j}$ can be rewritten as

$$
\sum_{l=j+1}^{j+k} c_{j+k-l}^{(d)} u_{l}+\sum_{l=1}^{j}\left(c_{j+k-l}^{(d)}-c_{j-l}^{(d)}\right) u_{l}=I_{1 j}^{(k)}+I_{2 j}^{(k)}, \quad \text { say. }
$$

It is readily seen that $I_{1 j}^{(k)}=\sum_{i=0}^{\infty} \psi_{i} \sum_{l=1}^{k} c_{k-l}^{(d)} \epsilon_{l+j-i}$. This, together with Hölder's inequality, implies that, for $d>1 / 2$,

$$
\begin{aligned}
E\left(I_{1 j}^{(k)}\right)^{2} & \leq \sum_{i=0}^{\infty}\left|\psi_{i}\right| \sum_{i=0}^{\infty}\left|\psi_{i}\right| E\left(\sum_{l=1}^{k} c_{k-l}^{(d)} \epsilon_{l+j-i}\right)^{2} \\
& \leq\left(\sum_{i=0}^{\infty}\left|\psi_{i}\right|\right)^{2} \sum_{l=0}^{k-1}\left(c_{l}^{(d)}\right)^{2} E \epsilon_{0}^{2} \leq C k^{\max \{2 d-1,0\}}
\end{aligned}
$$

Similarly, we have that, for $d>1 / 2$,

$$
E\left(I_{2 j}^{(k)}\right)^{2} \leq\left(\sum_{i=0}^{\infty}\left|\psi_{i}\right|\right)^{2} \sum_{l=0}^{j-1}\left(c_{k+l}^{(d)}-c_{l}^{(d)}\right)^{2} E \epsilon_{0}^{2} \leq C j^{\max \{2 d-3,0\}},
$$

where the last inequality follows from that, for any fixed $k$,

$$
\left|c_{k+l}^{(d)}-c_{l}^{(d)}\right| \leq C k l^{d-2}, \quad l=1,2, \cdots .
$$

It follows from (17)-(19) that, for $d>1 / 2$,

$$
\sum_{j=1}^{n-k} E\left(Z_{j+k}-Z_{j}\right)^{2} \leq 2 \sum_{j=1}^{n-k}\left\{E\left(I_{1 j}^{(k)}\right)^{2}+E\left(I_{2 j}^{(k)}\right)^{2}\right\} \leq C n^{\max \{1,2 d-2\}} .
$$

This implies (16) by Markov's inequality and noting $2 d-1>1$ for $d>1$. If $d=1$, then $Z_{j+k}-Z_{j}=\sum_{l=1}^{k} u_{l+j}, j \geq 1$, is a stationary linear process. (16) follows easily from the stationary ergodic theorem.

The proof of (16) is more laborious. Write $c_{l}^{*}=c_{l}^{(d)}-c_{l-1}^{(d)}, l \geq 0$ for convenience. By noting $1 / 2<d<1$ and $c_{0}^{*}=1,\left|c_{l}^{*}\right| \leq C l^{d-2}, l \geq 1$ from (20), we have that, for every $t \geq 1$,

$$
\sum_{l=0}^{\infty}\left|c_{l}^{*}\right| E\left|u_{t-l}\right| \leq\left(1+C \sum_{l=1}^{\infty} l^{d-2}\right) \sum_{k=0}^{\infty}\left|\psi_{k}\right| E\left|\epsilon_{0}\right|<\infty,
$$


and hence $\sum_{l=0}^{\infty} c_{l}^{*} u_{t-l}$ is well defined. Therefore, letting $c_{j}^{(d)}=0$ if $j<0$, simple algebra gives that $Z_{j+k}-Z_{j}$ is equal to

$$
\sum_{i=1}^{k} \sum_{l=0}^{\infty} c_{l}^{*} u_{j+i-l}-\sum_{i=1}^{k} \sum_{l=j+i}^{\infty} c_{l}^{*} u_{j+i-l}=I_{3 j}^{(k)}+I_{4 j}^{(k)}, \quad \text { say. }
$$

By Hölder's inequality, as in (18), it can be shown that

$$
\begin{aligned}
\sum_{j=1}^{n} E\left(I_{4 j}^{(k)}\right)^{2} & \leq k^{2} \sum_{j=1}^{n} \sum_{l=0}^{\infty}\left|c_{l+j}^{*}\right| \sum_{l=0}^{\infty}\left|c_{l+j}^{*}\right| E u_{-l}^{2} \\
& \leq C \sum_{j=1}^{n}\left(\sum_{l=j}^{\infty} l^{d-2}\right)^{2}=O\left(n^{2 d-1}\right)
\end{aligned}
$$

where we have used the bound: $E u_{t}^{2}=\sum_{k=0}^{\infty} \psi_{k}^{2} E \epsilon_{0}^{2}<\infty$. Similarly, we get that

$$
\sum_{j=1}^{n} E\left(I_{3 j}^{(k)}\right)^{2} \leq k^{2} \sum_{j=1}^{n}\left(\sum_{l=0}^{\infty}\left|c_{l}^{*}\right|\right)^{2} E u_{0}^{2}=O(n) .
$$

In terms of $(22),(23)$ and $\left(Z_{j+k}-Z_{j}\right)^{2}-\left(I_{3 j}^{(k)}\right)^{2}=I_{4 j}^{(k)}\left(2 I_{3 j}^{(k)}+I_{4 j}^{(k)}\right)$, it follows easily that

$$
\begin{aligned}
& \sum_{j=1}^{n} E\left|\left(Z_{j+k}-Z_{j}\right)^{2}-\left(I_{3 j}^{(k)}\right)^{2}\right| \\
\leq & \left\{\sum_{j=1}^{n} E\left(I_{4 j}^{(k)}\right)^{2}\right\}^{1 / 2}\left\{\sum_{j=1}^{n} E\left(2 I_{3 j}^{(k)}+I_{4 j}^{(k)}\right)^{2}\right\}^{1 / 2}=O\left(n^{d}\right)
\end{aligned}
$$

Recalling $1 / 2<d<1,(24)$ implies that $(1 / n) \sum_{j=1}^{n-k}\left[\left(Z_{j+k}-Z_{j}\right)^{2}-\right.$ $\left.\left(I_{3 j}^{(k)}\right)^{2}\right]=o_{P}(1)$. Now (16) follows if

$$
\frac{1}{n} \sum_{j=1}^{n-k}\left(I_{3 j}^{(k)}\right)^{2}-E\left(\sum_{i=1}^{k} \sum_{l=0}^{\infty} c_{l}^{*} u_{i-l}\right)^{2}=o_{P}(1) .
$$

Recalling that $u_{t}, t=0, \pm 1, \cdots$, is a stationary ergodic linear process, $I_{3 j}^{(k)}, j \geq 1$, still has the same properties as those of the process $u_{t}$. Therefore, (25) follows from the stationary ergodic theorem. This completes the proof of Proposition 1. 


\section{Conclusion}

This paper derives a functional limit theorem for general nonstationary fractionally integrated processes having no influence from prehistory. The results extend those given in Aknom and Gourieroux [1], Marinucci and Robinson [11] as well as Tanaka [16] to a more general model under weak conditions. By using established results, asymptotic distributions of sample autocovariances and sample autocorrelations based on these processes are also investigated. We mention that proofs of the main results in this paper are totally different from those in the literature, and the problem arises naturally in discussing fractionally integrated processes when the processes start at a given initial date.

\section{References}

1. J. Akonom, and C. Gourieroux. A functional central limit theorem for fractional processes. Discussion Paper \#8801. Paris: CEPREMAP, 1987.

2. H. J. Bierens. Higher-order sample autocorrelations and the unit root hypothesis. Journal of Econometrics 57:137-160, 1993.

3. P. Billingsley. Convergence of Probability Measures. New York: Wiley, 1968.

4. E. J. Hannan and C. C. Heyde. On limit theorems for quadratic functions of discrete time series. Ann. Math. Statist. 43:2056-2066, 1972.

5. U. Hassler. The sample autocorrelation function of $I(1)$ process. Statistical Papers 35:1-16, 1994.

6. U. Hassler. Sample autocorrelations of nonstationary fractionally integrated series. Statistical Papers 38:43-62, 1997.

7. D. P. Hasza. The asymptotic distribution of the sample autocorrelations for an integrated ARMA process. J. Amer. Stat. Assoc. 75:349-352, 1980.

8. J. R. M. Hosking. Asymptotic distributions of the sample mean, autocovariances and autocorrelations of long memory time series. Journal of Econometrics 73:261$284,1996$.

9. J. Komlós, P. Major and G. Tusnády. An approximation of partial sums of independent R.V.'s and sample DF. I. Z. Wahrsch. verw. Gebiete 32:111-131, 1975.

10. J. Komlós, P. Major and G. Tusnády. An approximation of partial sums of independent R.V.'s and sample DF. II. Z. Wahrsch. verw. Gebiete 34:33-58, 1976.

11. D. Marinucci, and P. M. Robinson. Alternative forms of fractional Brownian motion. Journal of Statistical Planning and Inference 80:13-23, 1999.

12. D. Marinucci and P. M. Robinson. Weak convergence of multivariate fractional processes. Stoch. Process. Appl. 86:103-120, 2000.

13. P. Newbold and C. Agiakloglou. Bias in the sample autocorrelations of fractional noise. Biometrika 80:698-702, 1993.

14. P. C. B. Phillips and V. Solo. Asymptotics for linear processes. Ann. Statist. 20:971-1001, 1992.

15. F. B. Sowell. The fractional unit root distribution. Econometrica 58:495-505, 1990.

16. K. Tanaka. The nonstationary fractional unit root. Econometric Theory 15:549$582,1999$. 
17. Q. Wang, Y-X. Lin and C. M. Gulati. Asymptotics for general fractionally integrated processes with applications to unit root tests. Econometric Theory, forthcoming 2003.

\section{Appendix 1: Proof of Theorem 1.}

For convenience, we always assume $c_{j}^{(d)}=0$, if $j<0$. Furthermore, we recall that $c_{j}^{(d)}=\Gamma(j+d) /\{\Gamma(d) \Gamma(j+1)\}$, for $j \geq 0$, and $\epsilon_{j}$ are i.i.d. random variables with $E \epsilon_{0}=0$ and $\sigma^{2}=E \epsilon_{0}^{2}<\infty$. The following facts (a) and (b) are well-known. The fact (c) can be found in Akonom and Gourieroux [1].

(a) If $E\left|\epsilon_{0}\right|^{\alpha}<\infty$, where $\alpha \geq 2$, then $\max _{-n \leq j \leq n}\left|\epsilon_{j}\right|=o_{P}\left(n^{1 / \alpha}\right)$.

(b) If $d>1 / 2$, then, $\left|c_{j}^{(d)}-\{\Gamma(d)\}^{-1} j^{d-1}\right| \leq C j^{d-2}$ for $j \geq 1$ and $\sum_{j=0}^{n}\left|c_{j}^{(d)}-c_{j-1}^{(d)}\right| \leq C n^{\max \{d-1,0\}}$, for $n \geq 1$.

(c) Assume that $\eta_{j}$ are independent $N(0,1)$ random variables. Then, for $d>1 / 2$,

$$
\frac{1}{n^{d-1 / 2}} \sum_{j=1}^{[n t]} c_{[n t]-j}^{(d)} \eta_{j} \Rightarrow \frac{1}{\Gamma(d)} B_{d}(t), \quad 0 \leq t \leq 1
$$

Using these facts, it can be shown that if $E\left|\epsilon_{0}\right|^{\max \{2,2 /(2 d-1)\}}<\infty$, then,

$$
\frac{1}{n^{d-1 / 2}} \sum_{j=1}^{[n t]} c_{[n t]-j}^{(d)} \epsilon_{j} \Rightarrow \frac{\sigma}{\Gamma(d)} B_{d}(t), \quad 0 \leq t \leq 1, \text { for } d>1 / 2 .
$$

Indeed, by applying Komlós, Major and Tusnády [9], [10], on a rich enough probability space, there exists a sequence of random variables $\eta_{j}, j \geq 1$, which are iid $N(0,1)$ such that, for $d>1 / 2$,

$$
\max _{1 \leq m \leq n}\left|\sum_{j=1}^{m} \epsilon_{j}-\sigma \sum_{j=1}^{m} \eta_{j}\right|=o_{P}\left(n^{\min \{(2 d-1) / 2,1 / 2\}}\right) .
$$

It follows from (c) that, for $d>1 / 2, n^{1 / 2-d} \sum_{j=1}^{[n t]} c_{[n t]-j}^{(d)} \eta_{j} \Rightarrow\{\Gamma(d)\}^{-1} B_{d}(t)$, $0 \leq t \leq 1$. On the other hand, $\sup _{0 \leq t \leq 1}\left|\sum_{j=1}^{[n t]} c_{[n t]-j}^{(d)} \epsilon_{j}-\sigma \sum_{j=1}^{[n t]} c_{[n t]-j}^{(d)} \eta_{j}\right|$ is bounded by $\sum_{j=0}^{n-1}\left|c_{j}^{(d)}-c_{j-1}^{(d)}\right| \max _{1 \leq m \leq n}\left|\sum_{k=1}^{m}\left(\epsilon_{k}-\sigma \eta_{k}\right)\right|$, which is $o_{P}\left(n^{d-1 / 2}\right)$ by using the fact (b) and (28). Therefore, (27) follows from a classical result (cf. Theorem 4.2 of Billingsley [3]). 
After these prelimilaries, we now give the proof of Theorem 1.

First it is shown that $(7)$ holds for $d \geq 1$. Let $C(B, l)=\sum_{k=0}^{l} \psi_{k} B^{k}$ and $C^{*}(B, l)=\sum_{i=0}^{l-1}\left(\sum_{k=i+1}^{l} \psi_{k}\right) B^{i}$, where $B$ is a backshift operator. From Lemma 2.1 in Phillips and Solo ( $[14])$, we have that $C(B, l)=$ $C(1, l)-C^{*}(B, l)(1-B)$. This implies that, for all $m \geq 1$ and $l \geq 1$,

$$
\begin{aligned}
& \sum_{j=1}^{m} c_{m-j}^{(d)} \sum_{k=0}^{l} \psi_{k} \epsilon_{j-k}=\sum_{j=1}^{m} c_{m-j}^{(d)} C(B, l) \epsilon_{j} \\
& +C(1, l) \sum_{j=1}^{m} c_{m-j}^{(d)} \epsilon_{j}-C^{*}(B, l) \sum_{j=1}^{m} c_{m-j}^{(d)}\left(\epsilon_{j}-\epsilon_{j-1}\right) .
\end{aligned}
$$

Therefore, we can write that, for all $m \geq 1$,

$$
\begin{gathered}
\sum_{j=1}^{m} c_{m-j}^{(d)} Z_{j} \sum_{j=1}^{m} c_{m-j}^{(d)}\left(\sum_{k=0}^{l} \psi_{k} \epsilon_{j-k}+\sum_{k=l+1}^{\infty} \psi_{k} \epsilon_{j-k}\right)+C(1, l) \sum_{j=1}^{m} c_{m-j}^{(d)} \epsilon_{j} \\
-C^{*}(B, l) \sum_{j=1}^{m} c_{m-j}^{(d)}\left(\epsilon_{j}-\epsilon_{j-1}\right)+\sum_{k=l+1}^{\infty} \psi_{k} \sum_{j=1}^{m} c_{m-j}^{(d)} \epsilon_{j-k} \\
=C(1, l) \sum_{j=1}^{m} c_{m-j}^{(d)} \epsilon_{j}+R_{1}(m, l)+R_{2}(m, l), \quad \text { say. }
\end{gathered}
$$

Since $C(1, l) \rightarrow b_{\psi}$, as $l \rightarrow \infty$, by using (27) and Theorem 4.2 of Billingsley [3], it suffices to show, for $d \geq 1$,

$$
\lim _{l \rightarrow \infty} \lim _{n \rightarrow \infty} P\left(\sup _{0 \leq t \leq 1}\left|R_{j}([n t], l)\right| \geq n^{d-1 / 2}\right)=0, \quad j=1,2 .
$$

It can be shown that, for all $m \geq 1, R_{1}(m, l)=\sum_{i=0}^{l-1}\left(\sum_{k=i+1}^{l} \psi_{k}\right) Y_{m, i}$, where $Y_{m, i}=c_{0}^{(d)} \epsilon_{m-i}-c_{m-1}^{(d)} \epsilon_{-i}+\sum_{j=1}^{m-1}\left(c_{m-j}^{(d)}-c_{m-j-1}^{(d)}\right) \epsilon_{j-i}$. Noting that $\max _{1 \leq m \leq n}\left|Y_{m, i}\right| \leq\left(2+\sum_{j=1}^{n-1}\left|c_{j}^{(d)}-c_{j-l}^{(d)}\right|\right) \max _{1 \leq j \leq n}\left|\epsilon_{j-i}\right|$, it follows from the facts (b) and (a) with $\alpha=2$ that, as $n \rightarrow \infty$,

$$
\sup _{0 \leq t \leq 1}\left|R_{1}([n t], l)\right| \leq C n^{d-1} \max _{-l \leq j \leq n}\left|\epsilon_{j}\right| \sum_{i=0}^{l-1} \sum_{k=i+1}^{l}\left|\psi_{k}\right|=o_{P}\left(n^{d-1 / 2}\right) \text {. }
$$


This proves (31) for $j=1$. To prove (31) for $j=2$, note that, for all $k \geq 1$,

$$
\begin{array}{r}
E \max _{1 \leq m \leq n}\left|\sum_{j=1}^{m} c_{m-j}^{(d)} \epsilon_{j-k}\right|=E \max _{1 \leq m \leq n}\left|\sum_{j=1}^{m} c_{m-j}^{(d)} \epsilon_{j}\right| \\
\leq \sum_{j=1}^{n}\left|c_{j}^{(d)}-c_{j-l}^{(d)}\right| E \max _{1 \leq j \leq n}\left|\sum_{i=1}^{j} \epsilon_{i}\right| \leq C n^{d-1 / 2},
\end{array}
$$

where the last inequality follows from the fact (b) and the well-known result: $E \max _{1 \leq j \leq n}\left|\sum_{i=1}^{j} \epsilon_{i}\right| \leq C n^{1 / 2}$. Hence, Markov's inequality implies that

$$
\begin{aligned}
& P\left(\sup _{0 \leq t \leq 1}\left|R_{2}([n t], l)\right| \geq n^{d-1 / 2}\right) \\
\leq & \frac{1}{n^{d-1 / 2}} \sum_{k=l+1}^{\infty}\left|\psi_{k}\right| E \max _{1 \leq m \leq n}\left|\sum_{j=1}^{m} c_{m-j}^{(d)} \epsilon_{j-k}\right| \leq C \sum_{k=l+1}^{\infty}\left|\psi_{k}\right| .
\end{aligned}
$$

Let $n \rightarrow \infty$ and then $l \rightarrow \infty$, we get (31) for $j=2$. The proof of (7), for $d \geq 1$, is now complete.

Next, the proof of ( 7 ), for $1 / 2<d<1$, is given. We still use (30), but here we choose $l=n$. Recalling $1 / 2<d<1$ and $E\left|\epsilon_{0}\right|^{2 /(2 d-1)}<\infty$, it follows from the facts (b) and (a) with $\alpha=2 /(2 d-1)$ that $\sum_{j=1}^{n}\left|c_{j}^{(d)}-c_{j-l}^{(d)}\right| \leq C$, and $\max _{-n \leq j \leq n}\left|\epsilon_{j}\right|=o_{P}\left(n^{d-1 / 2}\right)$. By noting $\sum_{k=0} k\left|\psi_{k}\right|<\infty$, similar to (32), we have that

$$
\sup _{0 \leq t \leq 1}\left|R_{1}([n t], n)\right| \leq C \max _{-n \leq j \leq n}\left|\epsilon_{j}\right| \sum_{i=0}^{n-1} \sum_{k=i+1}^{n}\left|\psi_{k}\right|=o_{P}\left(n^{d-1 / 2}\right) .
$$

On the other hand, similar to (33), we get that

$$
\begin{aligned}
P\left(\sup _{0 \leq t \leq 1}\left|R_{2}([n t], n)\right| \geq n^{d-1 / 2}\right) & \leq C n^{1-d} \sum_{k=n+1}^{\infty}\left|\psi_{k}\right| \\
& \leq C \sum_{k=n+1}^{\infty} k\left|\psi_{k}\right|=o(1) .
\end{aligned}
$$

These facts, together with (27) and Theorem 4.2 of Billingsley [3], imply (7) for $1 / 2<d<1$. This also completes the proof of Theorem 1 . 


\section{Appendix 2: A complementary proposition}

This proposition is used to show that the process $X_{t}$ defined by (1) with $X_{t}=0, t \leq 0$, is a special case of the process $Z_{t}$ defined by (5).

Proposition 2 Let $d>-1 / 2$,

$$
(1-B)^{d} Z_{t}=v_{t}, t=1,2, \cdots ; Z_{t}=0, t \leq 0,
$$

where $v_{t}, t \geq 1$ is an arbitrary well-defined process, $B$ is a Backshift operator and the fractional difference operator $(1-B)^{\gamma}$ is defined as in (2). Then, we have that $Z_{t}=0, t \leq 0$, and

$$
Z_{t}=\sum_{k=0}^{t-1} c_{k}^{(d)} v_{t-k}, \quad t=1,2, \cdots,
$$

where $c_{0}^{(0)}=1, c_{k}^{(0)}=0, k \geq 1$, and $c_{k}^{(\alpha)}=\Gamma(k+\alpha) /\{\Gamma(\alpha) \Gamma(k+1)\}, k \geq 0$, for $\alpha \neq 0,-1,-2, \cdots$.

Proof: At first, we assume that $d>-1 / 2$ and $d \neq 0,1,2, \cdots$. Under this assumption, we first show that

$$
c_{t}^{(d)}=-\sum_{k=1}^{t} c_{t-k}^{(d)} c_{k}^{(-d)}, \quad t=1,2, \cdots .
$$

Recalling $\Gamma(z+1)=z \Gamma(z)$ (for all $z \neq 0,-1, \cdots$, by definition), it is obvious that

$$
c_{1}^{(d)}=\frac{\Gamma(1+d)}{\Gamma(d) \Gamma(2)}=d, \quad c_{1}^{(-d)}=\frac{\Gamma(1-d)}{\Gamma(-d) \Gamma(2)}=-d .
$$

Hence, (37) holds for $t=1$. We next assume that (37) holds for $t=n$, i.e.,

$$
c_{t}^{(d)}=-\sum_{k=1}^{t} c_{t-k}^{(d)} c_{k}^{(-d)}, \quad t=1,2, \cdots, n .
$$

By induction, it suffices to show that

$$
c_{n+1}^{(d)}=-\sum_{k=1}^{n+1} c_{n+1-k}^{(d)} c_{k}^{(-d)}=-\sum_{k=0}^{n} c_{k}^{(d)} c_{n+1-k}^{(-d)} .
$$

To prove (39), by summing each term of (38), we obtain that

$$
\begin{gathered}
\sum_{t=1}^{n} c_{t}^{(d)}=-\sum_{t=1}^{n} \sum_{k=1}^{t} c_{t-k}^{(d)} c_{k}^{(-d)}=-\sum_{k=1}^{n} c_{k}^{(-d)} \sum_{t=k}^{n} c_{t-k}^{(d)} \\
=-\sum_{k=1}^{n} c_{k}^{(-d)} \sum_{t=0}^{n-k} c_{t}^{(d)}=-\sum_{k=0}^{n-1} c_{n-k}^{(-d)} \sum_{t=0}^{k} c_{t}^{(d)} .
\end{gathered}
$$


By using (10) and the definition of $c_{k}^{(\alpha)}$, it can be easily shown that, for all $k \geq 1$ and $\alpha \neq 0,-1,-2, \cdots$,

$$
\sum_{t=0}^{k} c_{t}^{(\alpha)}=c_{k}^{(1+\alpha)}=\frac{k+1}{\alpha} c_{k+1}^{(\alpha)} .
$$

In terms of (40), $c_{0}^{(d)}=1$ and (41) with $\alpha=d$, it follows that, for $d>-1 / 2$ and $d \neq 0,1,2, \cdots$,

$$
\frac{n+1}{d} c_{n+1}^{(d)}-1=-\frac{1}{d} \sum_{k=0}^{n-1}(k+1) c_{n-k}^{(-d)} c_{k+1}^{(d)}=-\frac{1}{d} \sum_{k=1}^{n} k c_{k}^{(d)} c_{n+1-k}^{(-d)} .
$$

On the other hand, (38) also implies that (recalling $c_{0}^{(d)}=c_{0}^{(-d)}=1$ )

$$
-c_{t}^{(-d)}=\sum_{k=0}^{t-1} c_{t-k}^{(d)} c_{k}^{(-d)}=\sum_{k=1}^{t} c_{k}^{(d)} c_{t-k}^{(-d)}, \quad t=1,2, \cdots, n .
$$

By summing each term of (43), it follows that

$$
-\sum_{t=1}^{n} c_{t}^{(-d)}=\sum_{k=1}^{n} c_{k}^{(d)} \sum_{t=0}^{n-k} c_{t}^{(-d)}
$$

In terms of (44), $c_{0}^{(-d)}=1$ and (41) with $\alpha=-d$, we obtain that, for $d>-1 / 2$ and $d \neq 0,1,2, \cdots$,

$$
\frac{n+1}{d} c_{n+1}^{(-d)}+1=\frac{1}{d} \sum_{k=1}^{n}(k-n-1) c_{k}^{(d)} c_{n+1-k}^{(-d)} .
$$

Now (39) follows immediately from summing the two sides of (42) and (45). This gives (37) by induction.

Because of (37), we can give the proof of Proposition 2 for $d>-1 / 2$ and $d \neq 0,1,2, \cdots$. Clearly, we only need to consider the case of $t \geq 1$. Recalling the definition of the fractional difference operator $(1-B)^{\gamma}$ (see (3)) and $Z_{t}=0, t \leq 0$, we rewrite (35) as

$$
\sum_{j=0}^{t-1} c_{j}^{(-d)} Z_{t-j}=v_{t}, \quad t=1,2, \cdots .
$$

It follows from (46) that, if $t=1$, then $Z_{1}=v_{1}$, i.e., (36) holds for $t=1$. Next we assume that (36) holds for $t=2, \cdots, n$, i.e.,

$$
Z_{j}=\sum_{k=0}^{j-1} c_{k}^{(d)} v_{j-k}, \quad j=1,2, \cdots, n
$$


By induction, it suffices to show that (47) also holds for $j=n+1$. To do this, we use (46) with $t=n+1$. In this case, recalling (47), (37) and $c_{0}^{(-d)}=1$, we have that

$$
\begin{gathered}
Z_{n+1}=v_{n+1}-\sum_{j=1}^{n} c_{j}^{(-d)} Z_{n+1-j}=v_{n+1}-\sum_{j=1}^{n} c_{j}^{(-d)} \sum_{k=0}^{n-j} c_{k}^{(d)} v_{n+1-j-k} \\
=v_{n+1}-\sum_{j=1}^{n} c_{j}^{(-d)} \sum_{k=j}^{n} c_{k-j}^{(d)} v_{n+1-k}=v_{n+1}-\sum_{k=1}^{n} v_{n+1-k} \sum_{j=1}^{k} c_{k-j}^{(d)} c_{j}^{(-d)} \\
=v_{n+1}-\sum_{k=1}^{n} c_{k}^{(d)} v_{n+1-k}=-\sum_{k=0}^{n=1} c_{k}^{(d)} v_{n+1-k} .
\end{gathered}
$$

This implies that (47) holds for $j=n+1$ and hence the proof of Proposition 2 is complete for $d>-1 / 2$ and $d \neq 0,1,2, \cdots$.

Next we show that Proposition 2 holds for $d=0,1,2, \cdots$. Recalling definition of $c_{k}^{(0)}, k \geq 0$, Proposition 2 is obvious for $d=0$. In the following, we assume that Proposition 2 holds for $d=m$. We will prove that (35) for $d=m+1$, i.e.,

$$
(1-B)^{m+1} Z_{t}=v_{t}, \quad t=1,2, \cdots ; Z_{t}=0, \quad t \leq 0 .
$$

It implies that $Z_{t} \sum_{k=0}^{t-1} c_{k}^{(m+1)} v_{t-k}, t=1,2, \cdots$, and then Proposition 2 follows by induction. To do this, let $Y_{t}=0, t \leq 0$, and $Y_{t}=(1-B) Z_{t}$, $t=1,2, \cdots$. It can be easily checked that $Z_{t}=\sum_{j=1}^{t} Y_{j}$ and, by using (49), $(1-B)^{m} Y_{t}=(1-B)^{m+1} Z_{t}=v_{t}, t=1,2, \cdots$. This implies that $Y_{t}$ satisfies (35) for $d=m$. By using the assumption that Proposition 2 holds for $d=m$, we obtain $Y_{t}=\sum_{k=0}^{t-1} c_{k}^{(m)} v_{t-k}, t=1,2, \cdots$, and therefore,

$$
\begin{aligned}
Z_{t} & =\sum_{j=1}^{t} Y_{j}=\sum_{j=1}^{t} \sum_{k=0}^{j-1} c_{k}^{(m)} v_{j-k}=\sum_{j=1}^{t} \sum_{k=1}^{j} c_{j-k}^{(m)} v_{k} \\
& =\sum_{k=1}^{t} v_{k} \sum_{j=k}^{t} c_{j-k}^{(m)}=\sum_{k=0}^{t-1} v_{t-k} \sum_{j=0}^{k} c_{j}^{(m)}=\sum_{k=0}^{t-1} v_{t-k} c_{k}^{(m+1)},
\end{aligned}
$$

$t=1,2, \cdots$, where we have used the well-known equality: for all $m \geq 1$, $\sum_{j=0}^{k} c_{j}^{(m)}=c_{k}^{(m+1)}, k=0,1,2, \cdots$. The proof of Proposition 2, for $d=$ $0,1,2, \cdots$, is now complete. 


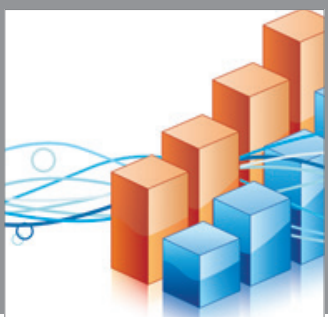

Advances in

Operations Research

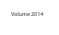

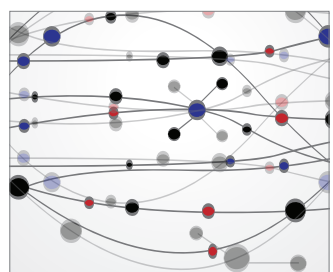

\section{The Scientific} World Journal
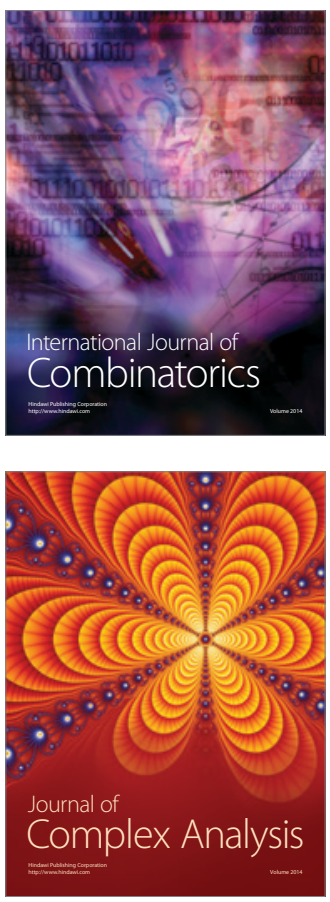

International Journal of

Mathematics and

Mathematical

Sciences
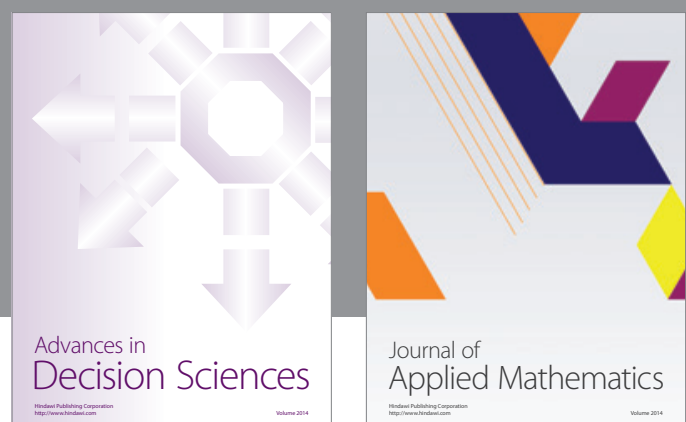

Journal of

Applied Mathematics
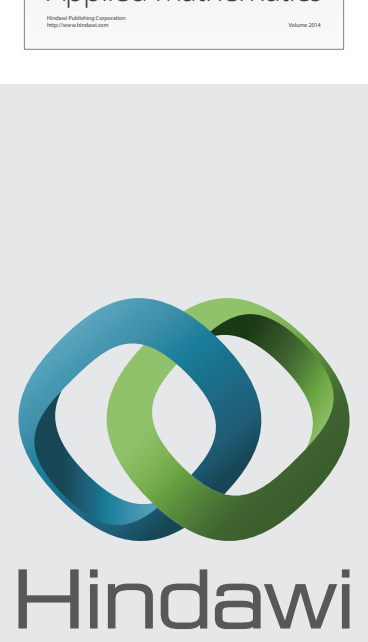

Submit your manuscripts at http://www.hindawi.com
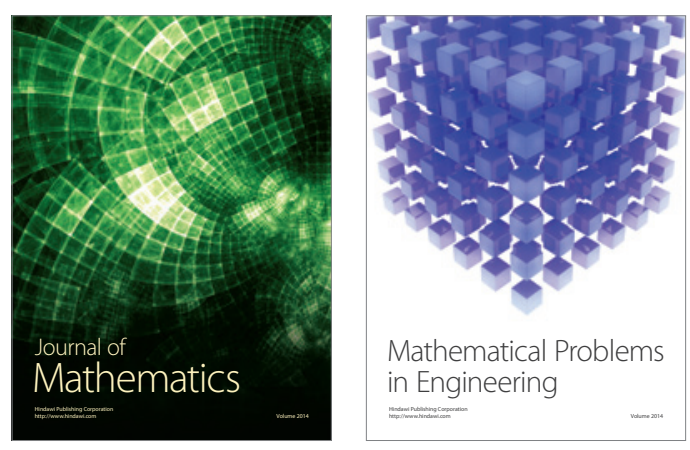

Mathematical Problems in Engineering
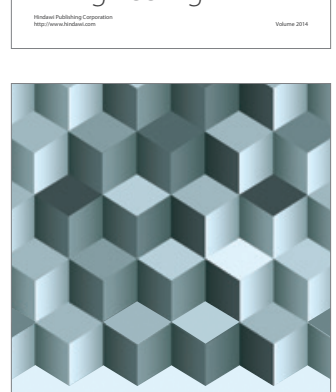

Journal of

Function Spaces
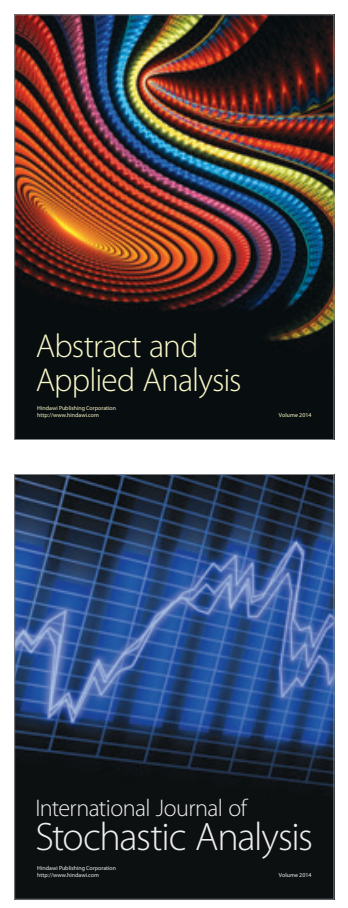

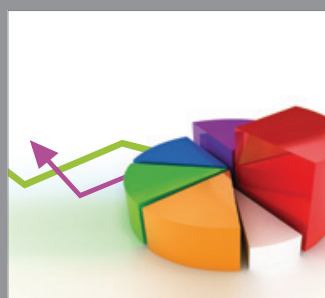

ournal of

Probability and Statistics

Promensencen
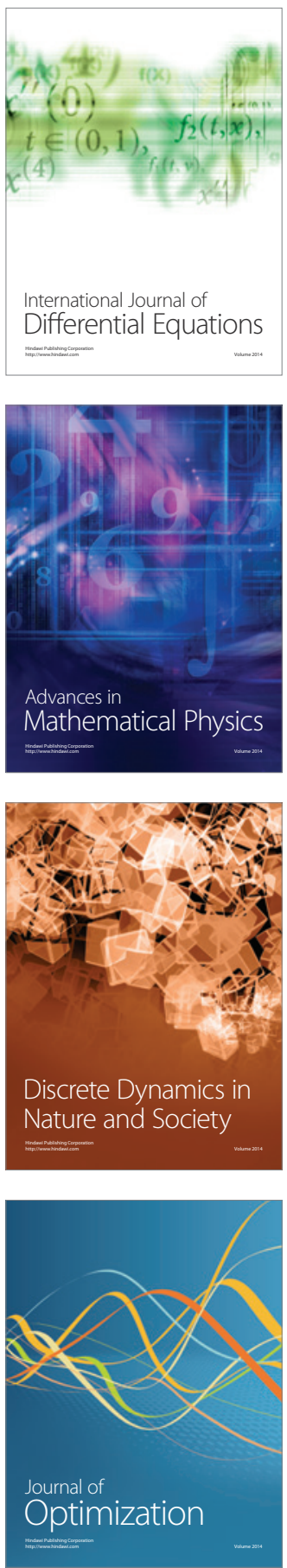